\title{
Colonic stenosis caused by infection of an intraperitoneal access port system: a rare complication of intraperitoneal chemotherapy for gastric cancer with peritoneal metastasis
}

\author{
Jun Kinoshita*, Sachio Fushida, Tomoya Tsukada, Katsunobu Oyama, Toshifumi Watanabe, Koichi Okamoto, \\ Isamu Makino, Keishi Nakamura, Hironori Hayashi, Hisatoshi Nakagawara, Tomoharu Miyashita, Hidehiro Tajima, \\ Hiroyuki Takamura, Itasu Ninomiya, Hirohisa Kitagawa, Takashi Fujimura and Tetsuo Ohta
}

\begin{abstract}
Background: Intraperitoneal (IP) chemotherapy is garnering attention as an effective treatment for gastric cancer with peritoneal metastasis. We report the case of a patient who developed colonic stenosis caused by infection of an IP access port system during IP chemotherapy. It was difficult to differentiate whether the extrinsic colonic stenosis arose from a catheter infection or peritoneal metastasis of the gastric cancer.

Case presentation: A 66-year-old Japanese man underwent total gastrectomy for gastric cancer. Because the intraoperative findings revealed peritoneal metastasis, a port system was implanted for subsequent IP chemotherapy. Two months after initiation of chemotherapy, he complained of vomiting and abdominal pain. A computed tomography scan revealed marked thickening of the sigmoid colon wall adjacent to the catheter of the IP access port system. A barium enema demonstrated extrinsic irregular stenosis of the sigmoid colon. Although it was difficult to distinguish whether infection or peritoneal metastasis had caused the colonic stenosis, we removed the port system to obtain a therapeutic diagnosis. Coagulase-negative staphylococci were detected by catheter culture. The wall thickening and stenosis of the sigmoid colon completely resolved after removal of the port system.

Conclusions: We report the case of a rare complication in association with an IP access port system. Infection of the port system should be considered as a differential diagnosis when colonic stenosis adjacent to the catheter is observed during IP chemotherapy.
\end{abstract}

Keywords: Intraperitoneal chemotherapy, Gastric cancer, Peritoneal metastasis, Port complication

\section{Background}

Gastric cancer is a major cause of cancer death worldwide. Recent advances in systemic chemotherapy regimens have shown encouraging tumor response rates and increased survival in patients with unresectable or metastatic gastric cancer [1]. However, treatment outcomes for patients with peritoneal metastasis, which is the most frequent metastatic pattern of recurrence, have not improved sufficiently [2].

\footnotetext{
*Correspondence: junkino0416@gmail.com

Department of Gastroenterologic Surgery, Division of Cancer Medicine,

Graduate School of Medical Science, Kanazawa University, 13-1 Takaramachi, Kanazawa 920-8641, Japan
}

Intraperitoneal (IP) chemotherapy is garnering attention as an effective treatment for peritoneal metastasis because of the theoretical advantage of higher local concentrations, prolonged tumor exposure, and reduced systemic toxicity [3,4]. IP chemotherapy was shown to prolong survival in a phase III study of ovarian cancer with peritoneal metastasis and has been approved as a recommended regimen by the National Cancer Institute in the United States [5]. IP chemotherapy has also been shown to be a promising treatment option for gastric cancer [6-9]. A multicenter randomized clinical trial is now ongoing to generate evidence regarding the effects 
of IP chemotherapy on gastric cancer with peritoneal metastasis.

A subcutaneous port and catheter system has been developed and is now the most common route through which chemotherapeutic agents are administered into the peritoneal cavity. The main advantages of subcutaneous systems are the low rate of port-related infections and the ease of drug administration into the peritoneal cavity [10]. However, complications associated with subcutaneous port systems have been reported in patients with ovarian cancer and gastric cancer [10-19].

We herein describe a patient who developed colonic stenosis caused by the infection of an IP access port system during IP chemotherapy for gastric cancer with peritoneal metastasis. It was difficult to differentiate whether the extrinsic colonic stenosis arose from a catheter infection or the peritoneal metastasis.

\section{Case presentation}

A 66-year-old Japanese male patient was discovered to have anemia (hemoglobin level, $6.1 \mathrm{~g} / \mathrm{dL}$ ) at a medical checkup. He underwent upper gastrointestinal endoscopy, which revealed Borrmann's type IV gastric cancer, and the biopsy findings resulted in a diagnosis of moderately differentiated adenocarcinoma. He then consulted with our hospital for surgical treatment.

A detailed examination revealed T3N1M0 (stage IIB) cancer according to the Union for International Cancer Control tumor, node, and metastasis classification. Although a laparotomy was performed for curative resection, serosal invasion of the primary tumor and multiple peritoneal metastases in the peritoneal cavity were discovered intraoperatively. Peritoneal lavage cytology was also positive according to the Japanese classification of gastric carcinoma. We performed a total gastrectomy for cytoreduction and implanted an IP access port system (Bardport-Ti; CR Bard Inc., New Jersey, United States) comprising of a titanium port with a bottom diameter of $31.47 \mathrm{~mm}$, a height of $14.5 \mathrm{~mm}$, and a 14.3- Fr singlelumen silicone catheter (Figure 1) for postoperative IP chemotherapy. The subcutaneous space was dissected and a pocket for implantation of the port was created in the right lower abdomen. A catheter was then inserted from the subcutaneous pocket into the peritoneal cavity, penetrating the abdominal wall, and the end of the catheter was placed in the pelvis.

The postoperative course was uneventful. On postoperative day 14 the patient began chemotherapy with S-1 (TS-1 ${ }^{\oplus}$; Taiho Pharmaceutical Company, Tokyo, Japan) at $80 \mathrm{mg} / \mathrm{m}^{2} /$ day ( 2 weeks on, 2 weeks off) and docetaxel administered intraperitoneally at $45 \mathrm{mg} / \mathrm{m}^{2}$ (days 1 and 15). He was discharged on postoperative day 29 and underwent outpatient chemotherapy.

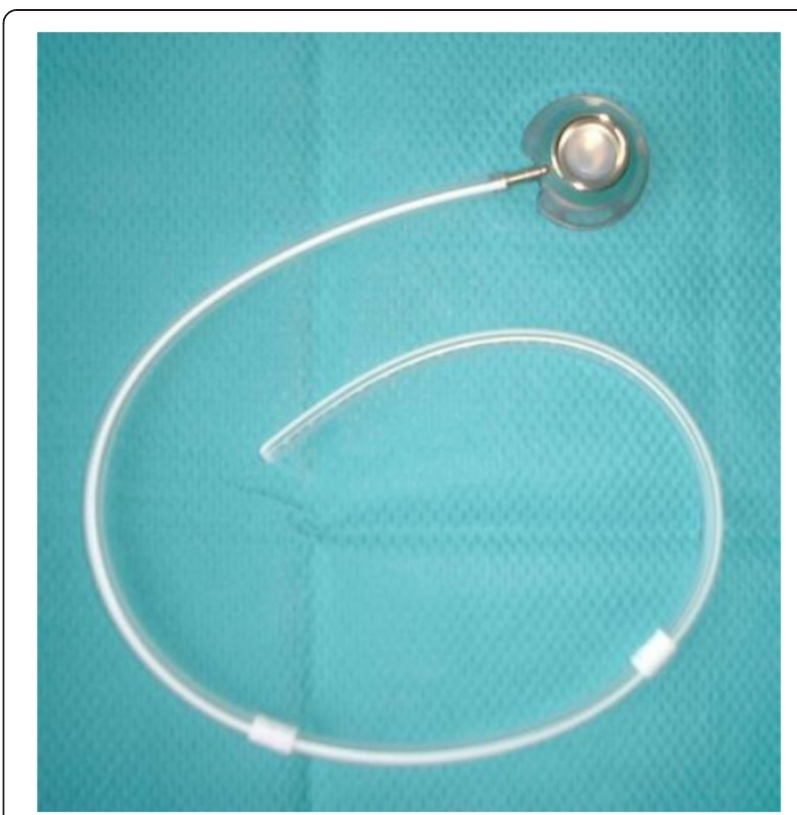

Figure 1 Intraperitoneal access port system. The intraperitoneal access port system comprised of a titanium port with a bottom diameter of $31.47 \mathrm{~mm}$, a height of $14.5 \mathrm{~mm}$, and a 14.3-Fr singlelumen silicone catheter.

Two months after implantation of the IP access port the patient developed vomiting and abdominal pain. An abdominal X-ray showed dilatation of the small intestine. He was alert upon admission to department of gastroenterologic surgery of our hospital, with a blood pressure of $110 / 53 \mathrm{mmHg}$, a pulse rate of 64 beats $/ \mathrm{min}$, and a body temperature of $36.8^{\circ} \mathrm{C}$. A physical examination was performed and localized tenderness was seen in the left lower abdomen apart from the port site. Neither port-site erythema nor swelling was observed. Laboratory data showed normal leukocyte and neutrophil counts $(8420 / \mu \mathrm{L}$ and $4910 / \mu \mathrm{L}$, respectively) and a slightly elevated C-reactive protein level $(3.6 \mathrm{mg} / \mathrm{dL})$. He was admitted with a diagnosis of ileus and a nasogastric tube was inserted. However, the left lower abdominal pain persisted.

Anabdominal computed tomography scan revealed marked wall thickening and narrowing of the lumen of the sigmoid colon adjacent to the catheter of the IP access port system (Figure 2A). A barium enema also demonstrated extrinsic irregular stenosis of the sigmoid colon (Figure 3A). We initially suspected peritoneal metastasis from gastric cancer and considered colostomy as a palliative operation. However, the stenosis was determined to have resulted from inflammation due to catheter infection because the intestinal stenosis was confined to the sigmoid colon, which was not a common site of peritoneal metastasis such as transverse-colon or rectum, and the 

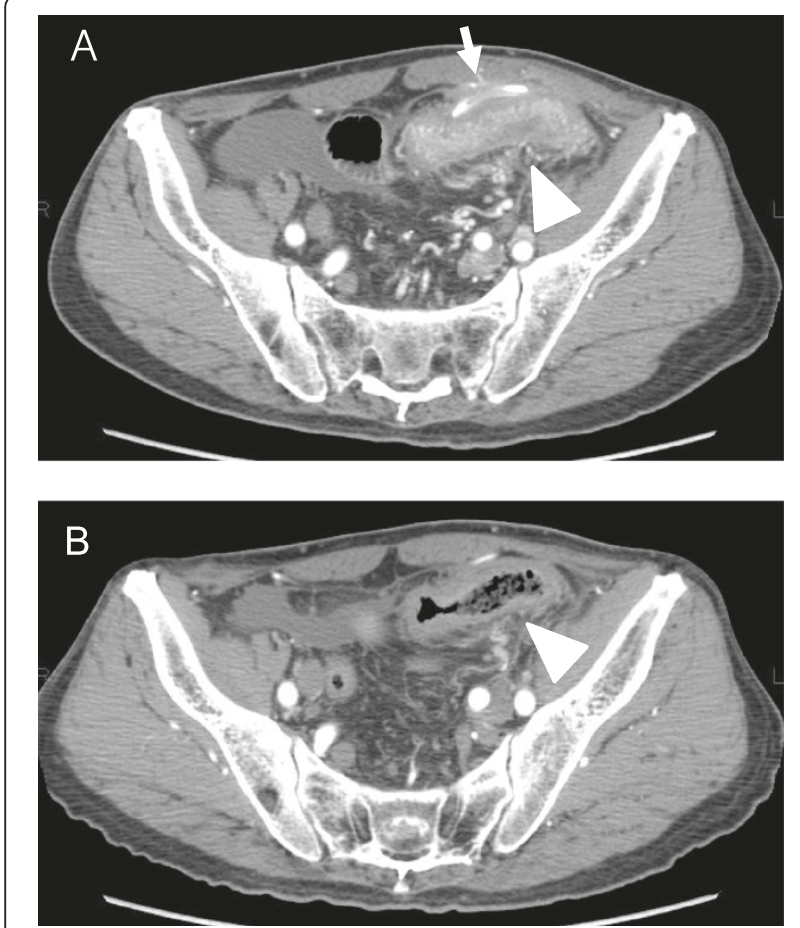

Figure 2 Computed tomographic changes in sigmoid colon before and after removal of intraperitoneal access port system. (A) A computed tomography scan showed marked wall thickening of the sigmoid colon (closed triangle) adjacent to the catheter (arrowhead) before removal. (B) Seven days after port removal, the wall thickening of the sigmoid colon had improved (open triangle). was no abscess in the subcutaneous space and no fibrin clots were occluding the lumen of the catheter.

However, after the port system removal the patient's abdominal pain immediately disappeared and he could ingest food normally several days later. In addition, an abdominal computed tomography scan demonstrated improvement in the thickening of the sigmoid colon wall seven days after port system removal (Figure 2B). Coagulase-negative staphylococci were detected by catheter culture. A colonofiberscopy showed no diverticula in the sigmoid colon, and that the mucosa was intact. Based on the patient's clinical course and the abovedescribed findings, we conclusively attributed the colonic stenosis to infection of the catheter. He was discharged on the 21 days after admission. The stenosis of the sigmoid colon had completely disappeared four months after port system removal as shown by a barium enema (Figure 3B).

Outpatient chemotherapy with $\mathrm{S}-1$ and intravenous docetaxel was performed. A re-laparoscopy was subsequently performed to evaluate the patient's response to chemotherapy. There were abnormal findings such as fibrosis, scar formation, or macroscopic progression of peritoneal metastasis in the sigmoid colon and its mesentery.

An IP access port system was re-implanted four months after removal. The patient remains alive without progression of peritoneal metastasis six months after surgery.

\section{Discussion}

A well-established principle of IP chemotherapy is the regional pharmacologic advantage achieved by direct instillation of drugs into the peritoneal cavity. A recent

phase II study of intravenous and IP paclitaxel combined usual course of peritoneal metastasis. Therefore, we removed the IP access port system under local anesthesia for a therapeutic diagnosis and began cephalosporin administration. At the time of port system removal, there

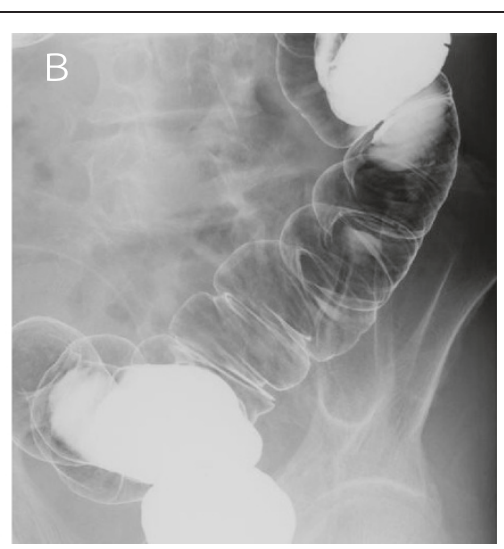

Figure 3 Extrinsic irregular stenosis of the sigmoid colon on barium enema. (A) Barium enema showed extrinsic irregular stenosis of the sigmoid colon around the catheter (arrowheads). (B) Four months after removal of the port system, the stenosis of the sigmoid colon had completely disappeared. 
with S-1 showed a 1-year overall survival rate of $78 \%$ and a median survival time of 22.5 months for patients with peritoneal metastasis from gastric cancer [6]. Other clinical trials involving IP chemotherapy with taxane agents have also shown favorable prognoses, with a median survival time of 16.2 to 24.6 months [7-9]. A multicenter randomized clinical trial is now ongoing to generate evidence regarding the effects of IP chemotherapy on gastric cancer with peritoneal metastasis.

To date, several reports have investigated complications associated with implanted subcutaneous ports and catheters for IP treatment of ovarian and gastric cancer [10-19]. Infection and malfunction were the most common complications; the rate of infection was reportedly $2.1 \%$ to $10.7 \%$, and the rate of malfunction was $3.7 \%$ to $18.2 \%$ (Table 1). Emoto et al. reported that intestinal bacteria were found in bacterial cultures of lavage fluid obtained through the port in six out of nine patients [19]. Although a relationship appears to exist between infection and gastrointestinal surgery performed concurrently with port implantation, no evidence of this relationship has been found in previous retrospective studies. In our patient, we implanted subcutaneous ports and catheters concurrently with total gastrectomy, but the catheter culture revealed coagulase-negative staphylococci. This suggests that the catheter infection was due to contamination of the port site at the time of needling or contamination via the infusion device because coagulase-negative staphylococci are the resident bacteria of the skin. It is important for medical staff members to take standard precautions to prevent port infections as presented here.

Previous studies have reported that gut-associated complications included fistula formation and perforation $(0.0$ to $3.5 \%)$. Braly et al. also reported one case of small bowel obstruction due to an IP catheter [17]. Fibrous sheath formation around the catheter was thought to pose a risk of small bowel obstruction [20].
To the best of our knowledge this is the first case of colonic stenosis as a complication of an IP port. We could not clarify the mechanism of the stenosis of the sigmoid colon in this case. We speculate that inflammation secondary to the catheter infection was the main contributor to the wall thickening and subsequent stenosis of the sigmoid colon because we did not observe numerous fibrous sheaths attached to the catheter at the time of removal. An important point of our case is that the extrinsic stenosis was remarkably similar in appearance to peritoneal metastasis. In general, peritoneal metastasis from gastric cancer frequently involves stenosis of the colorectum. In this case, the gut stenosis was not histologically confirmed by colonofiberscopy because it extended from the serosal side of the colonic wall. The findings obtained by barium enema were characteristic of the metastatic carcinoma that extended from the serosal side of the colonic wall.

Our patient had no fever and no severe acute inflammatory changes were found upon blood examination during the infectious episode. Therefore, it was difficult to distinguish whether infection or peritoneal metastasis caused the colonic stenosis. Such a diagnosis should be cautiously obtained because it substantially influences the subsequent treatment plan.

The occurrence of port complications in gastric cancer has been investigated in only one other report [19] according to our review of the literature, and has not been fully eliminated as is the case in ovarian cancer. Because peritoneal metastasis of gastric cancer more frequently involves enterostenosis than peritoneal metastasis of ovarian cancer, our case is thought to be significant in terms of the management of IP chemotherapy-induced complications, especially in patients with gastric cancer.

\section{Conclusions}

In summary, we report the case of a rare complication in association with an IP access port system. Infection of

Table 1 Complications associated with intraperitoneal access port systems in previous reports *n: number of patient

\begin{tabular}{|c|c|c|c|c|c|c|c|c|}
\hline Author & Year & ${ }^{*} n$ & $\begin{array}{l}\text { Type of } \\
\text { cancer }\end{array}$ & $\begin{array}{l}\text { Malfunction } \\
{ }^{*} \text { n (\%) }\end{array}$ & $\begin{array}{l}\text { Infection } \\
{ }^{*} \text { n (\%) }\end{array}$ & $\begin{array}{c}\text { Bowel perforation/fistula } \\
{ }^{*} \text { n (\%) }\end{array}$ & $\begin{array}{c}\text { Small bowel obstruction } \\
*^{*}(\%)\end{array}$ & $\begin{array}{c}\text { Total } \\
*_{\text {n }}(\%)\end{array}$ \\
\hline Pfeifle et al. [18] & 1984 & 54 & ovarian cancer & $3(5.5)$ & $3(5.5)$ & 0 & 0 & $6(11)$ \\
\hline Piccart et al. [12] & 1985 & 145 & ovarian cancer & $3(2.1)$ & $12(8.3)$ & $2(1.4)$ & 0 & $17(11.7)$ \\
\hline Braly et al. [17] & 1986 & 33 & ovarian cancer & $2(6.1)$ & $6(18.2)$ & $1(3.0)$ & $1(3.0)$ & $10(30.3)$ \\
\hline Davidson et al. [13] & 1991 & 227 & ovarian cancer & $20(8.8)$ & $12(5.3)$ & $8(3.5)$ & 0 & $40(17.6)$ \\
\hline Malmastorm et al. [14] & 1994 & 125 & ovarian cancer & $6(4.8)$ & $5(4.0)$ & 0 & 0 & $37(30)$ \\
\hline Topuz et al. [10] & 2000 & 56 & ovarian cancer & $6(10.7)$ & $3(5.4)$ & $1(1.8)$ & 0 & $10(18)$ \\
\hline Makhija et al. [15] & 2001 & 301 & ovarian cancer & $19(6.3)$ & $11(3.7)$ & 0 & 0 & $30(10)$ \\
\hline Walker et al. [16] & 2006 & 205 & ovarian cancer & $18(8.8)$ & $21(10)$ & $4(2.0)$ & 0 & $40(20)$ \\
\hline Emoto et al. [19] & 2012 & 131 & gastric cancer & $10(7.6)$ & $9(6.9)$ & $2(1.5)$ & 0 & $27(21)$ \\
\hline
\end{tabular}


the port system should be considered as a differential diagnosis when colonic stenosis adjacent to the catheter is observed during IP chemotherapy.

\section{Consent}

Written informed consent was obtained from the patient for publication of this case report and accompanying images. A copy of the written consent is available for review by the Editor-in-Chief of this journal.

\section{Abbreviation \\ IP: intraperitoneal.}

\section{Competing interests}

The authors declare that they have no competing interests.

\section{Authors' contributions}

JK wrote the case report. SF and TF interpreted the data related to the oncologic disease. JK, TT, TW, KoO, KN, and $\mathrm{KaO}$ performed the physical examination and medical care. IM, HH, HN, TM, H Tajima, H Takamura, IN, HK, and TO contributed to the writing and revision of the manuscript.

All authors read and approved the final manuscript.

Received: 28 February 2014 Accepted: 16 May 2014

Published: 4 June 2014

\section{References}

1. Siewert JR, Bottcher K, Roder JD, Busch R, Hermanek P, Meyer HJ: Prognostic relevance of systematic lymph node dissection in gastric carcinoma. German Gastric Carcinoma Study Group. Br J Surg 1993, 80:1015-1018

2. Allum WH, Powell DJ, McConkey CC, Fielding JW: Gastric cancer: a 25-year review. Br J Surg 1989, 76:535-540

3. Francis P, Rowinsky E, Schneider J, Hakes T, Hoskins W, Markman M: Phase I feasibility and pharmacologic study of weekly intraperitoneal paclitaxel: a gynecologic oncology group pilot study. J Clin Oncol 1995, 13:2961-2967.

4. Markman M, Brady MF, Spirtos NM, Hanjani P, Rubin SC, Gynecologic Oncology Group: Phase II trial of intraperitoneal paclitaxel in carcinoma of the ovary, tube, and peritoneum. J Clin Oncol 1998, 16:2620-2624.

5. Armstrong DK, Bundy B, Wenzel L, Huang HQ, Baergen R, Lele S, Copeland $\sqcup$, Walker JL, Burger RA, Gynecologic Oncology Group: Intraperitoneal cisplatin and paclitaxel in ovarian cancer. N Engl J Med 2006, 354:34-43.

6. Ishigami H, Kitayama J, Kaisaki S, Hidemura A, Kato M, Otani K, Kamei T, Soma D, Miyato H, Yamashita H, Nagawa H: Phase II study of weekly intravenous and intraperitoneal paclitaxel combined with S-1 for advanced gastric cancer with peritoneal metastasis. Ann Oncol 2010, 21:67-70.

7. Fushida S, Kinoshita J, Kaji M, Hirono Y, Goda F, Yagi Y, Oyama K, Sudo Y, Watanabe Y, Fujimura T: Society for study of peritoneal carcinomatosis in gastric cancer: phase I/II study of intraperitoneal docetaxel plus S-1 for the gastric cancer patients with peritoneal carcinomatosis. Cancer Chemother Pharmacol 2013, 71:1265-1272.

8. Fujiwara Y, Takiguchi S, Nakajima K, Miyata H, Yamasaki M, Kurokawa Y, Mori M, Doki Y: Intraperitoneal docetaxel combined with S-1 for advanced gastric cancer with peritoneal dissemination. J Surg Oncol 2012, 105:38-42.

9. Imano M, Yasuda A, Itoh T, Satou T, Peng YF, Kato H, Shinkai M, Tsubaki M, Chiba Y, Yasuda T, Imamoto H, Nishida S, Takeyama Y, Okuno K, Furukawa $H$, Shiozaki H: Phase II study of single intraperitoneal chemotherapy followed by systemic chemotherapy for gastric cancer with peritoneal metastasis. J Gastrointest Surg 2012, 16:2190-2196.

10. Topuz E, Saip P, Aydiner A, Salihoglu Y, Aydin Y, Topuzlu C: Catheter complications associated with intraperitoneal chemotherapy. Eur J Gynaecol Oncol 1998, 19:275-279.

11. Topuz E, Salihoglu Y, Aydiner A, Saip P, Tas F, Sozen T, Berkman S, Bengisu E: Celsite port and catheter as an intraperitoneal access device in the treatment of ovarian cancer. J Surg Oncol 2000, 74:223-226.
12. Piccart MJ, Speyer JL, Markman M, ten Bokkel Huinink WW, Alberts D, Jenkins J, Muggia F: Intraperitoneal chemotherapy: technical experience at five institutions. Semin Oncol 1985, 12:90-96.

13. Davidson SA, Rubin SC, Markman M, Jones WB, Hakes TB, Reichman B, Almadrones L, Chapman D, Lewis JL Jr, Hoskins WJ: Intraperitoneal chemotherapy: analysis of complications with an implanted subcutaneous port and catheter system. Gynecol Oncol 1991, 41:101-106.

14. Malmstrom H, Carstensen J, Simonsen E: Experience with implanted subcutaneous ports for intraperitoneal chemotherapy in ovarian cancer. Gynecol Oncol 1994, 54:27-34.

15. Makhija S, Leitao M, Sabbatini P, Bellin N, Almadrones L, Leon L, Spriggs DR, Barakat R: Complications associated with intraperitoneal chemotherapy catheters. Gynecol Oncol 2001, 81:77-81.

16. Walker JL, Armstrong DK, Huang HQ, Fowler J, Webster K, Burger RA, Clarke-Pearson D: Intraperitoneal catheter outcomes in a phase III trial of intravenous versus intraperitoneal chemotherapy in optimal stage III ovarian and primary peritoneal cancer: a gynecologic oncology group study. Gynecol Oncol 2006, 100:27-32.

17. Braly P, Doroshow J, Hoff S: Technical aspects of intraperitoneal chemo- therapy in abdominal carcinomatosis. Gynecol Oncol 1986, 25:319-333.

18. Pfeifle CE, Howell SB, Markman M, Lucas WE: Totally implantable system for peritoneal access. J Clin Oncol 1984, 2:1277-1280.

19. Emoto $S$, Ishigami $H$, Hidemura $A$, Yamaguchi $H$, Yamashita $H$, Kitayama J, Watanabe T: Complications and management of an implanted intraperitoneal access port system for intraperitoneal chemotherapy for gastric cancer with peritoneal metastasis. Jpn J Clin Oncol 2012, 42:1013-1019.

20. Alberts DS, Markman M, Armstrong D, Rothenberg ML, Muggia F, Howell SB: Intraperitoneal therapy for stage III ovarian cancer: a therapy whose time has come! J Clin Oncol 2002, 20:3944-3946.

doi:10.1186/1477-7819-12-177

Cite this article as: Kinoshita et al:: Colonic stenosis caused by infection of an intraperitoneal access port system: a rare complication of intraperitoneal chemotherapy for gastric cancer with peritoneal metastasis. World Journal of Surgical Oncology 2014 12:177.

\section{Submit your next manuscript to BioMed Central and take full advantage of:}

- Convenient online submission

- Thorough peer review

- No space constraints or color figure charges

- Immediate publication on acceptance

- Inclusion in PubMed, CAS, Scopus and Google Scholar

- Research which is freely available for redistribution 